\title{
Applications of photonic crystal fibers in optical communications - What is in the future?
}

Bjarklev, Anders Overgaard; Lin, Chinlon

Published in:

The 18th Annual Meeting of the IEEE Lasers and Electro-Optics Society, 2005. LEOS 2005.

Link to article, DOI:

10.1109/LEOS.2005.1548256

Publication date:

2005

Document Version

Publisher's PDF, also known as Version of record

Link back to DTU Orbit

Citation (APA):

Bjarklev, A. O., \& Lin, C. (2005). Applications of photonic crystal fibers in optical communications - What is in the future? In The 18th Annual Meeting of the IEEE Lasers and Electro-Optics Society, 2005. LEOS 2005. IEEE. https://doi.org/10.1109/LEOS.2005.1548256

\section{General rights}

Copyright and moral rights for the publications made accessible in the public portal are retained by the authors and/or other copyright owners and it is a condition of accessing publications that users recognise and abide by the legal requirements associated with these rights.

- Users may download and print one copy of any publication from the public portal for the purpose of private study or research.

- You may not further distribute the material or use it for any profit-making activity or commercial gain

- You may freely distribute the URL identifying the publication in the public portal 


\title{
Applications of Photonic Crystal Fibers in Optical Communications ---What is in the Future?
}

\author{
Anders Bjarklev \\ Research Center COM, Technical University of Denmark, DTU Building 345v, DK-2800, \\ Kgs.Lyngby,Denmark (e-mail: ab@com.dtu.dk) \\ Chinlon Lin \\ Center for Advanced Research in Photonics (CARP), The Chinese University of Hong Kong, \\ Shatin, NT, Hong Kong (e-mail: chinlon@ie.cuhk.edu.hk)
}

\begin{abstract}
:
Superior control of guiding properties in photonic-crystal fibers led to several interesting applications in optical communications ranging from nonlinear optical signal processing to high-power fiber amplifiers. This paper will review recent developments and discuss the future possibilities.
\end{abstract}

\section{Summary:}

Over the past decade, a tremendous development has taken place within the area of optical components having a full or partly periodic structure incorporated. Such artificial optical materials are often called photonic crystals, because they form an optical equivalent to the electronic crystals in semiconductors, and they appear both as key elements in novel optical fibres and in integrated optical devices. The most mature class of components are the optical fibres (often named photonic crystal fibres, microstructured fibres, or holey fibres). This new class of optical waveguides is characterized by a carefully engineered cross-section of glass (or polymer) riddled with a pattern of miniature holes. Introducing these micron-scale air-glass structures gives the fibre a wide range of unique optical properties, including novel light-guiding mechanisms, and enhanced nonlinearity [1-2]. The field of integrated optical waveguides may also benefit strongly from the development of this technology, and we may envision that scientific progress over the next decade will lead to several orders of magnitude improvement in the amount of possible optical components on an integrated optical chip.

The use of microstructures in optical fibres have opened new developments in various areas of fiber applications, and it is interesting that each of these areas actually takes advantage of different aspects of the enhanced physical performance enabled by the location of microstructures in the fibers. One of the highly interesting possibilities of the photonic crystal fibers lies in the ability to confine light in a very small cross section area through the use of high index contrast between holes and glass. This is used in the so-called highly nonlinear photonic crystal fibers HNL-PCFs [2]. The opposite possibility is explored in the case of socalled large-mode-area photonic crystal fibers, LMA-PCFs, which moves the nonlinear limitations to higher power levels by spreading of light to larger areas than possible in conventional fibers.

The possibility of microstructuring does, however, hold further interesting possibilities, and one is to obtain very high numerical apertures. When these possibilities are combined in an optimal manner, one of the most promising applications of photonic crystal fibers appears in the rapidly developing area of compact highpower doped PCF fiber lasers and amplifiers [3]. These may have primary applications in areas such a highpower laser-based material processing and biomedical applications, but will also find unique applications within optical communications, including, for example, broadband video distribution in the cable TV's hybrid-fiber-coax (HFC) networks or free-space optical communications.

In future optical networks one of the enabling technologies is tunable component elements or subsystem modules including reconfigurable routers, switches etc. Thus, the development of a technology platform that allows construction of tuning components is critical. Lately, photonic bandgap fibers, filled with liquid crystals, have proven to be candidates for such a platform. Photonic bandgap fibers offer unique wave-guiding properties that are strongly related to the design of the air holes in the cladding of the fiber. These wave- 
guiding properties may be altered by filling the air holes with a material, for example a liquid crystal that changes optical properties when subjected to, for example, an optical or an electrical field [4]. The utilization of these two basic properties allows design of tuneable optical devices for reconfigurable optical networks. In this presentation, we will focus on applications of such devices and discuss recent results.

Among the very interesting topics within the research on photonic crystal fibers are the possibilities for tailoring the waveguide dispersion in these devices. This is already used in the design of dispersion controlled highly nonlinear PCFs [2]. Several applications of these dispersion-flattened high-nonlinearity photonic crystal fibers for optical communications have been demonstrated in nonlinear optical signal processing, including wideband tunable wavelength conversion, nonlinear signal conditioning and reshaping, optical signal regeneration, ultrashort optical pulse compression, etc. [5-8].

With the design flexibility and the ability to tailor the dispersion properties of these microstructured PCFs it may also be envisioned that better and more efficient dispersion compensating elements can be developed through the use of very high dispersion values obtainable in PCFs. Such PCF based DCF (dispersion compensating fiber) or DCM (dispersion-compensating module) may replace the current DCFs in future highcapacity DWDM systems for long-haul and metro networks.

One question is whether the transmission loss of the PCFs can be as low as current silica SMF so they can be used as the basic long-distance optical signal transmission medium. The recent research results indicated loss as low as $0.3 \mathrm{~dB} / \mathrm{km}$ has been achieved and wideband DWDM system experiment has been demonstrated [9], so the potential for using PCF for telecom-level transmission seems to be there. However, given the fact that current worldwide fiber networks used SMF of various varieties and were already well built and installed (and with much over-capacity at the present), even if PCFs can achieve very low loss transmission, their use as transmission medium will most likely be only for special niche applications [9], but not in any significant scale. On the other hand, recent research results indicated that using hole-assisted structured, "holey fibers" with much more bending tolerance (low loss even at a very small bending radius) have been developed and deployed in NTT's FTTH network to alleviate the FTTH fiber cable installation problems at home [10].

This talk will review these and other PCF applications in optical communications and discuss what may be in the future.

\section{References:}

1. A. Bjarklev, "Photonic crystal fibers: fundamentals to emerging applications," (Tutorial) CMM1, CLEO '05, May 2005, Baltimore, Maryland, USA.

2. K. P. Hansen, J. R. Folkenberg C. Peucheret and A. Bjarklev, "Fully dispersion controlled triangular-core nonlinear photonic crystal fiber," OFC 2003 Postdeadline Paper PD2-1, Atlanta, Georgia, March 2003.

3. Richard E. Kennedy and J. R. Taylor, "All fiber, integrated, kilowatt level subpicosecond chirped pulse Yb amplification system using an air-core photonic Bandgap fiber," CMM1, CLEO '05, May 2005, Baltimore, Md., USA.

4. M. W. Haakestad, T. T. Larsen, M. D. Nielsen, H. E. Engan and A. Bjarklev, "Electrically tunable fiber device based on a nematic liquid crystal filled photonic Bandgap fiber, "ECOC'04 Postdeadline Paper Th.4.3.2, Sept. 2004, Stockholm, Sweden.

5. K. K. Chow, C. Shu, Chinlon Lin and A. Bjarklev, "Polarization-insensitive widely tunable wavelength converter based on four-wave mixing in a dispersion-flattened nonlinear photonic crystal Fiber," IEEE Photonics Technology Letters, vol. 17, p. 624, March 2005.

6. K. K. Chow, C. Shu, Chinlon Lin, and A. Bjarklev, "All-optical signal restoration by spectral filtering of self-phase modulation in nonlinear photonic crystal fiber," in Proc. ECOC '04, Paper We4.P.104, Sept. 2004, Stockholm, Sweden.

7. K. K. Chow, C. Shu, Chinlon Lin, and A. Bjarklev, "All-optical pulse compression and reshaping by spectral filtering from self-phase modulation in a nonlinear photonic crystal fiber," CLEO '05, May 2005, Baltimore, Maryland, USA.

8. Zhaoxin Wang, Chinlon Lin, K. K. Chow, Yuen-Ching Ku, and Anders Bjarklev, "Nonlinear suppression of interferometric crosstalk with dispersion imbalanced loop mirror using dispersion flattened high-nonlinear photonic crystal fiber,' CLEO '05, May 2005, Baltimore, Maryland, USA.

9. Kazuhide Nakajima et al., "Ultra wide band $190 \mathrm{~Gb} / \mathrm{s}$ WDM transmission over a long length and low loss PCF," OFC'04, Postdeadline Paper PDP 23, Feb. 2004, Los Angeles, California.

10. H. Shinohara, "Broadband Expansion in Japan", Plenary Talk, OFC'05, March 2005, Anaheim, California. 